\title{
Respecting Human Dignity through Individualized Care
}

\section{Evridiki Papastavrou*}

Assistant Professor, Department of Nursing, School of Health Sciences, Cyprus University of Technology, Cyprus

"To see the humanbeing behind the disease as an ultimately self-determining individual with potentialities within himself" is the utmost expression of respect to dignity. This philosophical perspective of nursing is described in Lanara's book Heroism as a Nursing Value [1], as it is influenced by the Greek literature and also discussed by contemporary nurse philosophers [2]. Although dignity is recognized as a core issue in the Universal Declaration of Human Rights [3] and as a central nursing value within the International Code of Ethics [4] and national nursing codes, the concept is still vague leading to confusion about its meaning. Jacelon et al. [5]'s identification of behavioral characteristics of dignity make an important contribution to science by facilitating the development of an operational definition that lends itself to the development of nursing interventions that promote patient dignity $[5,6]$. Operationalizing dignity as well as the implications for everyday practice, Gallagher et al. [7] support that much of what is described as contributing to dignity in care could be grouped under the heading of individualised care. It is also interesting to note that the code for the standards of conduct, performance and ethics for nurses and midwives of the Nursing and Midwifery Council of UK [8] sets as the very first point the following: "make the care of people your first concern, treating them as individuals and respecting their dignity". Combining philosophical, conceptual and empirical work, Gallagher organizes published work in 4 common themes including the environment of care that sets the context and the conditions for the preservation of dignity as well as the staff attitudes and behavior in treating the patient as an individual. The third theme described as "culture of care" was supported by references to holistic and individualised care and participation in an atmosphere that respects individual differences. The fourth theme was related to a wide range of specific care activities that have the potential to promote or ruin dignity, supporting that attention to the preferences of the individual patients also points to the importance of dignity of identity. In a later study [9] the authors support that nurses should find out what matters for the dignity of individuals by developing skills for understanding individuals' needs. Much of empirical work that explores individualised nursing care has been done by Suhonen et al. [10] who developed the individualized care model that has been found to capture attributes which characterize individualized care. The model highlights the importance of patients' clinical situation, personal life situation and decisional control as predictors of individualized care.

A concept analysis for preserving dignity in caring for older adults [11] describes individualized care as the first of the defining attributes of the concept. It also supports that to be cared for in the way which older people prefer, in line with individual needs and preferences, preserves dignity. Similarly, "seeing the patient as a unique person" was one of the main themes found by Hall and Høy [12] in re-establishing dignity through their exploration of nurses experiences of caring for older hospital patients. The authors stress that patients should not be seen as a diagnosis but cared for as an individual with unique beliefs, values, capabilities and life histories. However assisting patients in maintaining dignity is not always achievable owing to restricted time, lack of resources and priority of symptom management. Professional nurses face the challenge to focus on the identification of individual needs and preferences and on patients own perspective and interpretation of dignity and respect.

Even though dignity is a multidimensional construct and gaps exist in nurses understanding of the concept, the aforementioned studies have provided sufficient information to focus on Operationalizing respect for dignity in nursing practice.

\section{References}

1. Lanara VA (1996) Heroism as a Nursing Value: A Philosophical Perspective (2nd edn), G Papanikolaou SA Graphic Arts, Greece.

2. Nordenfelt L (2004) The Varieties of Dignity. Health Care Anal 12: 69-81.

3. The Universal Declaration of Human Rights.

4. ICN (2006) The ICN Code of Ethics for Nurses. ICN, Geneva

5. Jacelon CS, Connelly TW, Brown R, Proulx K, Vo T (2004) A concept analysis of dignity for older adults. J Adv Nurs 48: 76-83.

6. Jacelon CS, Dixon J, Knafl KA (2009) Development of the Attributed Dignity Scale. Res Gerontol Nurs 2: 202-213.

7. Gallagher A, Li S, Wainwright P, Jones IR, Lee D (2008) Dignity in the care of older people - a review of the theoretical and empirical literature. BMC Nurs 7: 11.

8. Standards of Conduct, Performance and Ethics for Nurses and Midwived.

9. Baillie L, Gallagher A (2011) Respecting dignity in care in diverse care settings: Strategies of UK nurses. Int J Nurs Pract 17: 336-341.

10. Suhonen R, Valimaki M, Leino-Kilpi H, Katajisto J (2004) Testing the individualized care model. Scand J Caring Sci 18: 27-36.

11. Anderberg P, Lepp M, Berglund AL, Segesten K (2007) Preserving dignity in caring for older adults: a concept analysis. J Adv Nurs 59: 635-643.

12. Hall EO, Hoy B (2012) Re-establishing dignity: nurses' experiences of caring for older hospital patients. Scand J Caring Sci 26: 287-294.

*Corresponding author: Evridiki Papastavrou, Assistant Professor, Department of Nursing, School of Health Sciences, Cyprus University of Technology, Cyprus, E-mail: e.papastavrou@cut.ac.cy

Received May 21, 2012; Accepted May 23, 2012; Published May 25, 2012

Citation: Papastavrou E (2012) Respecting Human Dignity through Individualized Care. J Nurs Care 1:e104. doi:10.4172/2167-1168.1000e104

Copyright: (C) 2012 Papastavrou E, et al. This is an open-access article distributed under the terms of the Creative Commons Attribution License, which permits unrestricted use, distribution, and reproduction in any medium, provided the original author and source are credited. 